\title{
Surgery for retrosternal goiter: cervical approach
}

\author{
Xu Wang ${ }^{1,2 \#}$, Yuqiu Zhou ${ }^{2 \#}$, Chao $\mathrm{Li}^{2}$, Yongcong Cai ${ }^{2}$, Tianqi $\mathrm{He}^{1,2}$, Ronghao Sun ${ }^{2}$, Wen Tian $^{3}$, \\ Zhengqi Tang ${ }^{4}$ Jianfeng Sheng ${ }^{5}$, Dingrong Liu ${ }^{6}$, Chunhan Gui ${ }^{2}$, Dingfen Zeng ${ }^{2}$, Chunyan Shui ${ }^{2}$, \\ Jian Jang', Guiquan Zhu' ${ }^{2}$, Yudong Ning ${ }^{2}$, Wei Wang ${ }^{2}$
}

${ }^{1}$ Department of Clinical Medicine, Chengdu Medical College, Chengdu 610041, China; ${ }^{2}$ Department of Head and Neck Surgery, Sichuan Cancer Hospital \& Institute, Sichuan Cancer Center, School of Medicine, University of Electronic Science and Technology of China, Chengdu 610041, China; ${ }^{3}$ Department of General Surgery, The General Hospital of the People's Liberation Army, Beijing 100853, China; ${ }^{4}$ Department of Otolaryngology Head and Neck Surgery, Zigong Third People's Hospital, Zigong 643000, China; Department of Otorhinolaryngology Head and Neck Surgery, Mianyang Third People's Hospital, Mianyang 621000, China; ${ }^{6}$ Department of Otorhinolaryngology Head and Neck Surgery, Neijiang Second People's Hospital, Neijiang 100191, China

Contributions: (I) Conception and design: X Wang, C Li, Y Zhou, R Sun; (II) Administrative support: C Li, R Sun; (III) Provision of study materials or patients: Y Cai, T He, C Gui, D Zeng, C Shui; (IV) Collection and assembly of data: J Jang, G Zhu, Y Ning, W Wang; (V) Data analysis and interpretation: W Tian, Z Tang, J Sheng, D Liu; (VI) Manuscript writing: All authors; (VII) Final approval of manuscript: All authors.

\#These authors contributed equally to this work.

Correspondence to: Chao Li; Ronghao Sun. Department of Head and Neck Surgery, Sichuan Cancer Hospital \& Institute, Sichuan Cancer Center, School of Medicine, University of Electronic Science and Technology of China, Chengdu 610041, China.

Email: headneck@qq.com; Sunronghao@qq.com.

Backgroundk Retrosternal goiter refers to when the thyroid gland extends from the neck to the substernal portion, descending below the thoracic inlet into the mediastinum. It is typically accompanied by compressive symptoms, and most patients need to undergo surgery. This retrospective study set out to analyze the surgical approach to retrosternal goiter and to evaluate perioperative complications, with the aim of recommending best surgical technique.

Methods: We carried out retrospective analysis of 115 patients with retrosternal goiter treated at our center between May 4, 2011 and March 19, 2019. We analyzed patient characteristics, surgical methods, and perioperative complications using SPSS.

Results: Of the 115 patients in our study, 112 underwent thyroidectomy by cervical approach, with only 3 requiring an extracervical approach. The median age of the patients was 52.3 years, and the majority were female $(81.74 \%)$. Most of the patients (73.91\%) experienced no symptoms but were diagnosed with tracheal compression during surgery or preoperative imaging examination. Ninety-eight $(85.22 \%)$ of our patients underwent preoperative evaluation of their condition by CT imaging. No obvious surgical contraindications were found before thyroid function tests. The mean operation time was $115.11 \mathrm{~min}$, and the average amount of bleeding during surgery was $54.43 \mathrm{~mL}$. The mean postoperative hospital stay was 5.38 days. In 109 cases (94.78\%), the goiter was found to be benign, and malignancy was diagnosed in 6 patients $(5.22 \%)$. Of the 112 patients who were treated with the cervical approach, 7 (6.25\%) experienced recurrent laryngeal nerve palsy; 6 of these cases were transitory and 1 was permanent. The number of patients treated by cervical and extracervical approach who experienced transient hypocalcaemia was 23 (20.54\%) and 2, respectively. Transient hypoparathyroidism affected 16 patients (14.29\%) treated by cervical approach. Two patients had tracheomalacia phenomenon and one patient had pleural effusion after surgery. No cases experienced permanent hypocalcemia, permanent hypoparathyroidism, postoperative hematoma, tracheostomy, or death.

Conclusions: Retrosternal goiter surgery is challenging for surgeons. The best surgical approach for the patient should be based on CT scan evaluation. In our study, based on preoperative CT imaging and inoperation evaluation, $50 \%$ of the tumor volume was located below the thoracic inlet and $50 \%$ of the tumor volume was located above the thoracic inlet in almost all of the patients. Both sections could be successfully removed via a cervical incision, and no obvious complications were observed during the perioperative 
period. With careful planning and execution before surgery and meticulous operation during surgery, most retrosternal goiters can be safely treated by cervical approach.

Keywords: Retrosternal goiter; thyroid; surgery

Submitted Jun 05, 2019. Accepted for publication Mar 05, 2020.

doi: 10.21037 /gs.2020.03.43

View this article at: http://dx.doi.org/10.21037/gs.2020.03.43

\section{Introduction}

Multinodular goiter (MNG) is defined as an enlarged thyroid gland with multiple nodules. Ultrasound-based screenings have shown MNG to have an incidence of about $10 \%$ (1). In the majority of cases, MNG is caused by iodine deficiency, which leads to excessive secretion of thyroidstimulating hormone (TSH). Retrosternal, substernal, also known as substernal or intrathoracic goiters are a subgroup of MNG, the common characteristics of which is the thyroid gland extending below the thoracic entrance into the mediastinum $(2,3)$.

Previous studies have reported retrosternal goiters to compress the surrounding structures, causing airway compression, difficulty swallowing, obstruction of the superior vena cava, and hoarseness (4). Surgery is the treatment of choice for retrosternal goiters, and typical reasons for surgery include compression of the surrounding structures, malignant risk, and intrathoracic extension (1). Most retrosternal goiters can be surgically removed through a cervical incision alone, although in some specific cases, manubriotomy or sternotomy incision may be required $(4,5)$. Previous studies have shown factors such as recurrent goiters, obstruction of the superior vena cava, malignant thyroid tumors, and the depth of tumor invasion in the aortic arch to increase the chance of a thoracic surgical approach being taken $(5,6)$. In these cases, patients need specific and careful preoperative evaluation and specialized surgical management, as well as observation for any postoperative complications. The purpose of this article is to sum up our center's experience in treating patients with retrosternal goiter.

\section{Methods}

A total of 115 patients ( 21 males and 94 females; mean age, 52.29 years) who were preoperatively diagnosed with retrosternal goiter and underwent thyroidectomy at the Department of Head and Neck Surgery and Thoracic
Surgery, Sichuan Cancer Hospital were enrolled in this retrospective study. Patients underwent a functional study with baseline thyroid function tests, parathyroid hormone (PTH), flexible fibreoptic laryngoscopy (FNE), and neck ultrasound. Of the patients, 98 (85.22\%) underwent preoperative CT examination to facilitate preoperative surgical planning. CT examination allowed the size, location, density of the goiter behind the sternum, and the relationship with the trachea, esophagus and surrounding large blood vessels to be determined. FNE was performed preoperatively to assess vocal cord mobility in each patient. If symptoms such as hoarseness and dysphagia (mainly with liquids) were present, the one-sided recurrent laryngeal nerve was considered to be damaged. Levels of serum calcium and PTH were measured before and after the operation.

\section{Results}

Between May 4, 2011 to March 19, 2019, 115 patients with a preoperative diagnosis of retrosternal goiter underwent thyroidectomy at our center. Cervical approach was used with 112 patients, with the remaining 3 patients requiring sternotomy. Patient characteristics are summarized in Table 1.

The median age of the patients was 52.29 years, and $81.74 \%$ were female. Eighty-five (73.91\%) patients were diagnosed with tracheal compression during surgery or preoperative imaging examination. No obvious surgical contraindications were found before thyroid function tests. Before the operation, 98 (85.22\%) of the patients underwent CT evaluation. Based on CT scan image analysis, we found the largest tumor volume to be $20.0 \mathrm{~cm}$ $\times 14.0 \mathrm{~cm}$, with the goiter extending beyond the aortic arch and $50 \%$ of the tumor volume located below the thoracic inlet. The preoperative CT images are summarized in Figure 1 . The main purposes of the surgery were to relieve compression symptoms, improve appearance, and remove 
potential malignant thyroid tumors.

As shown in Table 2, $12(10.43 \%)$ of the patients underwent total thyroidectomy, with the rest undergoing non-total thyroidectomy (keeping part of the gland). The mean operation time was $115.11 \mathrm{~min}$, and the amount of bleeding during surgery was $54.43 \mathrm{~mL}$. The average postoperative hospital stay was 5.38 days. The goiter was found to be benign in 109 cases (94.78\%) and malignant in the remaining 6 cases $(5.22 \%)$. Postoperatively, hoarseness and dysphagia (mainly with liquids) was experienced temporarily by $6(5.36 \%)$ patients and permanently by 1 (0.89\%) patient.

Temporary hypocalcemia affected 23 (20.54\%) patients treated by cervical approach and $2(2 / 3)$ patients who

Table 1 Patient characteristics

\begin{tabular}{lc}
\hline Characteristics & $\mathrm{N}=115$ \\
\hline Age (year) & 52.29 \\
Gender & \\
Male & $21(18.26 \%)$ \\
Female & $94(81.74 \%)$ \\
Tracheal compression & $85(73.91 \%)$ \\
Previous thyroid surgery & $4(3.48 \%)$ \\
Preoperative CT & $98(85.22 \%)$ \\
\hline
\end{tabular}

underwent sternotomy. Temporary hypoparathyroidism was experienced by 16 patients (14.29\%) treated by cervical approach. Two patients had tracheomalacia phenomenon and one patient had pleural effusion after surgery. None of the patients underwent tracheostomy, or experienced permanent hypocalcemia, permanent hypoparathyroidism, postoperative hematoma, tracheostomy, or death. The incidences of perioperative period complications are summarized in Table 3.

\section{Discussion}

Numerous definitions of retrosternal goiter have been proposed, including: (I) the goiter extending more than 3 $\mathrm{cm}$ below the thoracic inlet; (II) at least $50 \%$ of the goiter being located in the chest; or (III) the gland extending to below the thoracic entrance into the mediastinum $(3,4,6,7)$. In this study, we used the extension of the mass below the level of the thoracic inlet as the criterion for retrosternal goiter $(8,9)$.

After a mass at the superior mediastinum is revealed by chest X-ray, or when the lower edge of the mass exceeds the plane of the thoracic entrance margin, retrosternal goiter can be identified by using CT or MRI scan to accurately measure the proportions of the gland present in the neck and chest.

Retrosternal goiter usually changes slowly. Therefore,
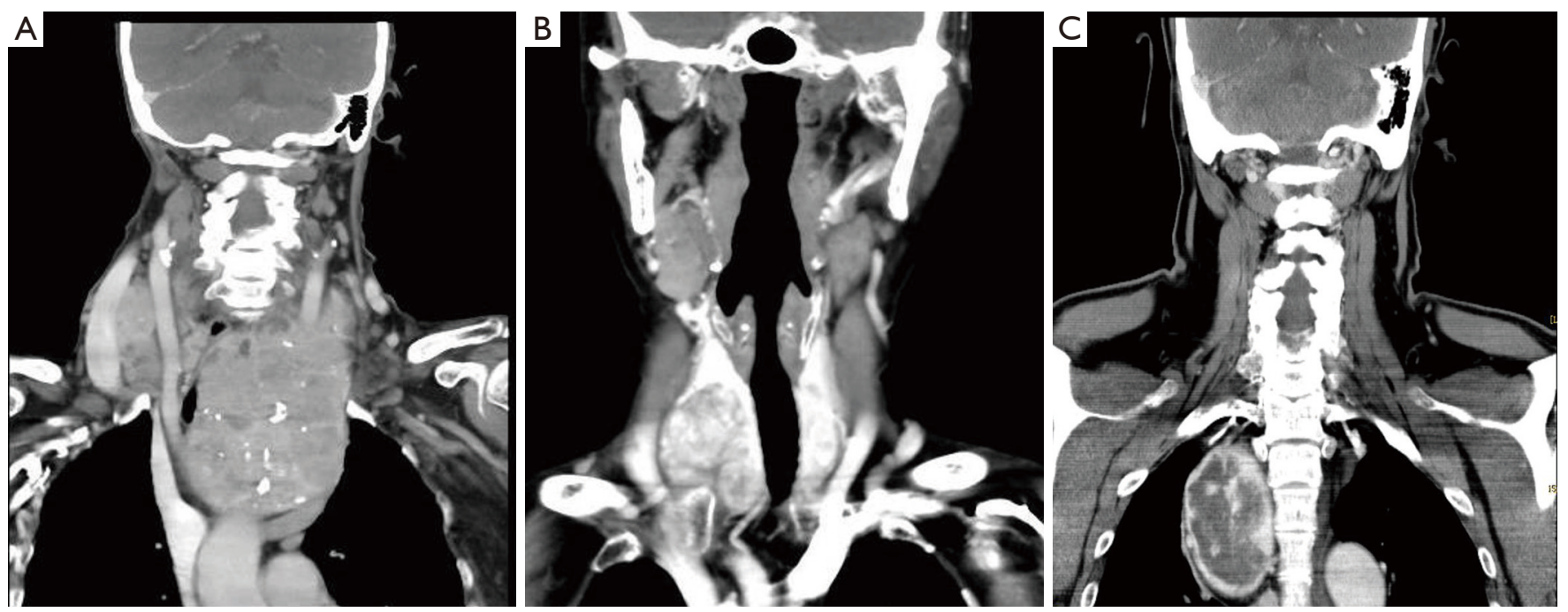

Figure 1 Based on the preoperative CT imaging examination, in most of the patients, 50\% of the tumor volume was located: (A) below the thoracic inlet; (B) above the thoracic inlet. (C) After the lesion thyroid was removed in this patient, the upper mediastinal mass was not revealed from the neck. 
Table 2 Surgical information

\begin{tabular}{lc}
\hline Variable & $\mathrm{N}=115$ \\
\hline Approach & $112(97.39 \%)$ \\
Cervical & $3(2.61 \%)$ \\
Thoracic & 115.11 \\
Operation time (min) & 54.43 \\
Blood loss (mL) & \\
Operative method & $12(10.43 \%)$ \\
Total thyroidectomy & $103(89.57 \%)$ \\
Non-total thyroidectomy & \\
Final pathology & $109(94.78 \%)$ \\
Benign & $6(5.22 \%)$ \\
Malignant & 5.38 \\
Median length of hospital stay (day)
\end{tabular}

patients often do not experience obvious clinical symptoms. Surgery is the treatment of choice for symptomatic retrosternal goiter (dyspnea, dysphagia, or superior vena cava syndrome) (10). The main reasons for surgery are: (I) most retrosternal goiters tend to grow and can compress adjacent structures; (II) there is a $15 \%$ incidence of malignancy (4); and (III) more than $95 \%$ of cases the goiter can be removed by a cervical approach (11).

A total of 115 patients who were diagnosed with retrosternal goiter were included in our study. Of these, $85(73.91 \%)$ were diagnosed with tracheal compression during surgery or preoperative imaging examination. A review of the CT and neck ultrasound of patients revealed that, in most cases, $50 \%$ of the tumor volume was located below the thoracic inlet and $50 \%$ of the tumor volume was located above the thoracic inlet (Figure 1A,B). The largest tumor volume was $20.0 \mathrm{~cm} \times 14.0 \mathrm{~cm}$, with more than $50 \%$

Table 3 Perioperative complications after surgery

\begin{tabular}{|c|c|c|}
\hline Perioperative complication & \multicolumn{2}{|c|}{ Incision (N=115) } \\
\hline \multicolumn{3}{|c|}{ Recurrent laryngeal nerve palsy } \\
\hline Temporary & $6(5.36 \%)$ & 0 \\
\hline Permanent & $1(0.89 \%)$ & 0 \\
\hline \multicolumn{3}{|l|}{ Hypoparathyroidism } \\
\hline Temporary & $16(14.29 \%)$ & 0 \\
\hline Permanent & 0 & 0 \\
\hline \multicolumn{3}{|l|}{ Hypocalcemia } \\
\hline Temporary & $23(20.54 \%)$ & $2(66.67 \%)$ \\
\hline Hematoma & 0 & 0 \\
\hline Hemorrhage & 0 & 0 \\
\hline Pneumothorax & 0 & 0 \\
\hline Pleural effusion & 0 & $1(33.33 \%)$ \\
\hline Tracheomalacia & $2(1.79 \%)$ & 0 \\
\hline Tracheostomy & 0 & 0 \\
\hline Mortality & 0 & 0 \\
\hline
\end{tabular}



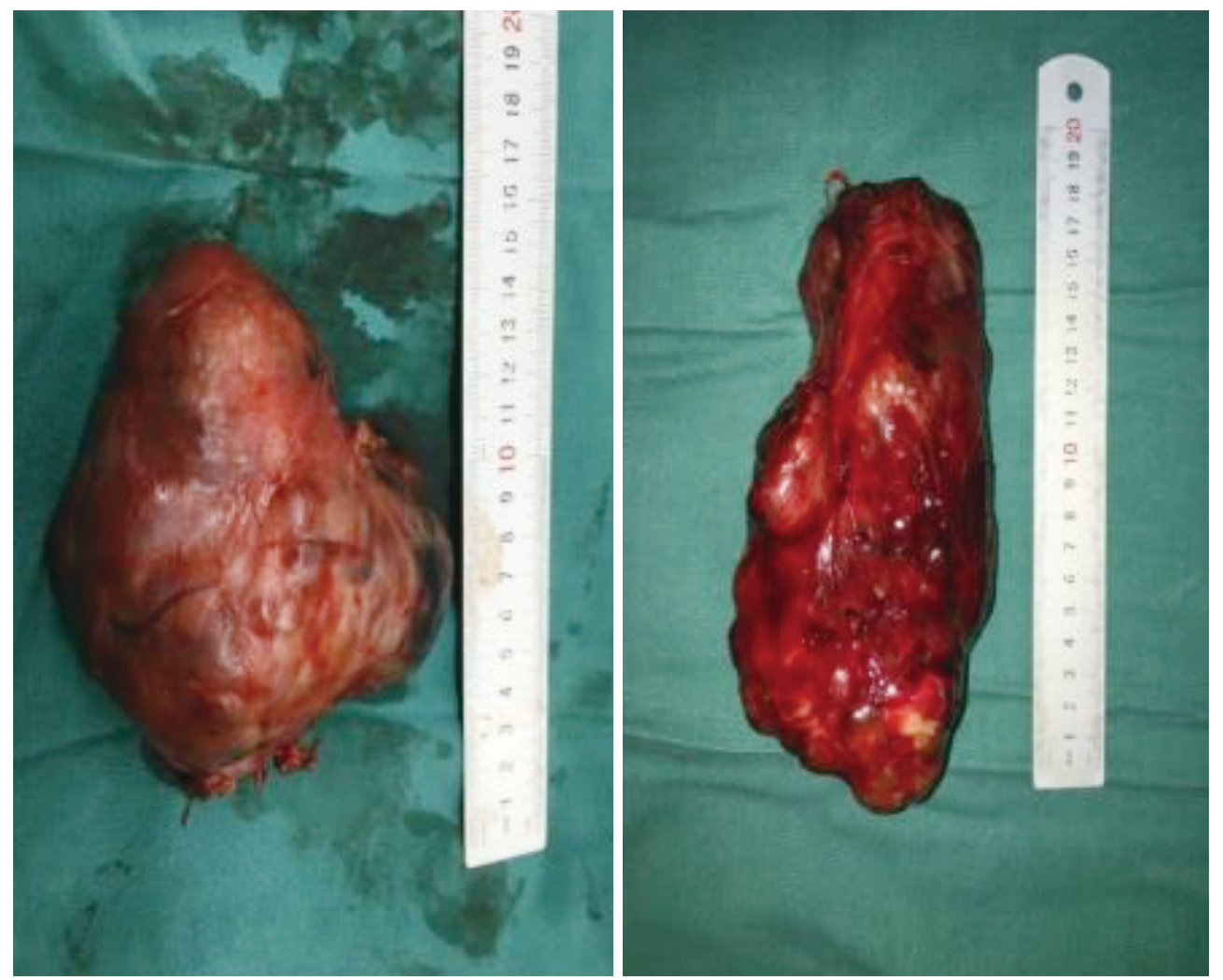

Figure 2 The largest tumor measured $20.0 \mathrm{~cm} \times 14.0 \mathrm{~cm}$.

below the thoracic entrance. CT imaging showed the tumor to extend beyond the aortic arch, and the innominate artery, left common carotid artery, and left subclavian artery to be obviously compressed (Figures 1A,2).

According to previous reports, choosing an extracervical approach has often depended on the CT scan-based depth of goiter expansion in the mediastinum and extension beyond the aortic arch $(2,12)$. Multiple studies have also recommended CT as the most valuable radiological examination for evaluating retrosternal goiter because it can assess the location of the mass, the sternum to the mediastinum, and the anatomical relationship between adjacent structures (5,13-15).

Studies have shown that fine needle aspiration (FNA) biopsy of thyroid nodules with ultrasound positioning has a higher predictive power (16). However, FNA is not advisable because the needle is inserted behind the sternum, and ultrasound cannot fully reveal the surrounding structures deep inside the mediastinum. The incidence of benign retrosternal goiters is also relatively high, and so preoperative FNA is not recommended (12).
In our study, there were 98 (85.22\%) patients who were preoperatively examined by CT. Chest X-ray and ultrasound provides us with preliminary radiological evidence of the progression of the goiter to the superior mediastinum. Followed by CT examination, they can show the tracheal compression caused by the mass (lumen stenosis or skew) and the relationship with the surrounding tissue.

Cervical approach was used for 112 cases in our study, with the remaining 3 cases removed by extracervical approach. The largest tumor, as mentioned above, was successfully removed in an operation lasting 170 minutes. The patient experienced some bleeding $(200 \mathrm{~mL})$ and stayed in hospital for a period of 5 days following surgery. No serious perioperative complications occurred. The tumor embodied the "iceberg" type described by Simó to be a factor in taking a cervical approach (2).

Our experience emphasizes the importance of head reclination in terms of surgical position. When the cervical approach is chosen, the shoulder should be raised with the head back to fully expose the surgical field. Then, using blunt separation combined with meticulous operation to 
identify and protect important blood vessels, nerves, and the parathyroid glands, the glands should be gradually pulled out of the mediastinum into the neck; this is the key to successfully removing the mass from the neck.

There were 3 patients in our study who had their tumor removed using an extracervical approach. In the first case, the patient's lesion was located in the lower thyroid of the right lobe, which was deep into the superior mediastinum. When the surface vein of the thyroid was separated, there was a large amount of bleeding, which proved difficult to stop, and so sternotomy had to be performed mid-surgery. The operation was successful and the recurrent laryngeal nerve and parathyroid glands were both preserved. In the case of the second patient, after the thyroid lesion was removed, the upper mediastinal mass was not revealed from the neck. The upper mediastinal thyroid was therefore removed by thoracotomy (Figure 1C). For the third patient, who was first seen in thoracic surgery, the preoperative CT examination revealed an anterior mediastinal tumor. Therefore, sternotomy was performed to remove the tumor, during which the mediastinal pleura ruptured, resulting in postoperative pleural effusion.

According to previous reports, another reason for a thoracic approach is a malignant thyroid tumor $(17,18)$. If there is evidence of a locally advanced malignancy, some believe cervical thyroidectomy combined with a thoracic approach can achieve the best curative outcome (12). In our study, there were $6(5.22 \%)$ patients diagnosed with a malignancy. All 6 of these patients had their tumor removed using a cervical approach. Some authors have also found that secondary or even multiple operations increase adhesion to surrounding tissues, and tight adhesions make dissection through cervical access difficult, thus increasing the likelihood of requiring an extracervical approach (19). In our study, 4 (3.48\%) patients with recurrent goiters did not require an extracervical approach. Local adhesion, the recurrent laryngeal nerve, and the abnormal position of the parathyroid gland caused by the first operation all contribute to the difficulty of a second operation. Four of the patients in our study had recurrent goiter in this group. Due to the enlarged mass encountered during operation, the surrounding tissue was pressed to form a clear gap and the adhesion of the original surgical site was found to be mostly located in the neck and contralateral glands, and the goiters were smoothly removed by cervical approach.

Voice changes following thyroid surgery are common. Subjective changes in sound without significant nerve damage occur in $30 \%$ to $87 \%$ of patients (20).
Recurrent laryngeal nerve palsy suggests locally invasive thyroid malignancy. In contrast, recurrent laryngeal nerve palsy caused by benign MNGs or ectopic thyroid tissue accounts for only $1 \%$ of cases (21). When symptoms like voice (hoarseness) appear, the recurrent laryngeal nerve is considered to be damaged. Bilateral recurrent laryngeal nerve palsy can be associated with acute airway obstruction and the need for tracheostomy or other airway widening procedures (20). Some of the main reasons for recurrent laryngeal nerve injury reasons are: (I) the normal route of the RLN has been altered by a space-occupying lesion, with a greater impact on the right side than on the left $(12,22)$; (II) the recurrent laryngeal nerve has spread to the posterior surface of the goiter; or (III) bilateral cervical goiter (23). The reported incidence of temporary and permanent RLN palsy after thyroid operations is $9.8 \%$ and $2.3 \%$, respectively (24). In comparison, the incidence of temporary and permanent RLN palsy in our patients was 5.36\% (6 patients) and $0.89 \%$ (1 patient), respectively. In the 6 cases of temporary RLN , the recurrent laryngeal nerve showed fully during the operation, and nerve monitoring during the resection was used to detect nerve signals, and all cases showed good signal.

Postoperative transient hoarseness is thought to be associated with neurological edema caused by mechanical damage during cervical lymph node dissection. In the case of one particular patient, the tumor was wrapped around the right recurrent laryngeal nerve and could not be completely dissected and separated. The right recurrent laryngeal nerve was therefore cut and recurrent laryngeal nerve anastomosis was performed at the same time. The contralateral nerve signal was normal. During the operation, this patient was found to have symptoms of tracheomalacia, so tracheal suspension was adopted in the operation. No serious complications such as dyspnea occurred after operation.

Bilateral recurrent laryngeal nerve palsy can be associated with acute airway obstruction and tracheostomy or other airway widening procedures (20). The position of the recurrent laryngeal nerve changes due to compression of the surrounding tissue structure by the retrosternal goiter. This is also the reason why the incidence of postoperative recurrent laryngeal nerve injury is higher than with average thyroid surgery. Some previous studies have recommended avoiding blind manipulation and using a transthoracic approach, which offers better surgical field exposure for the lesion area, thus helping to avoid serious complications $(7,25)$. Surgeons should be familiar with the anatomy of the recurrent laryngeal nerve. With better exposure during 


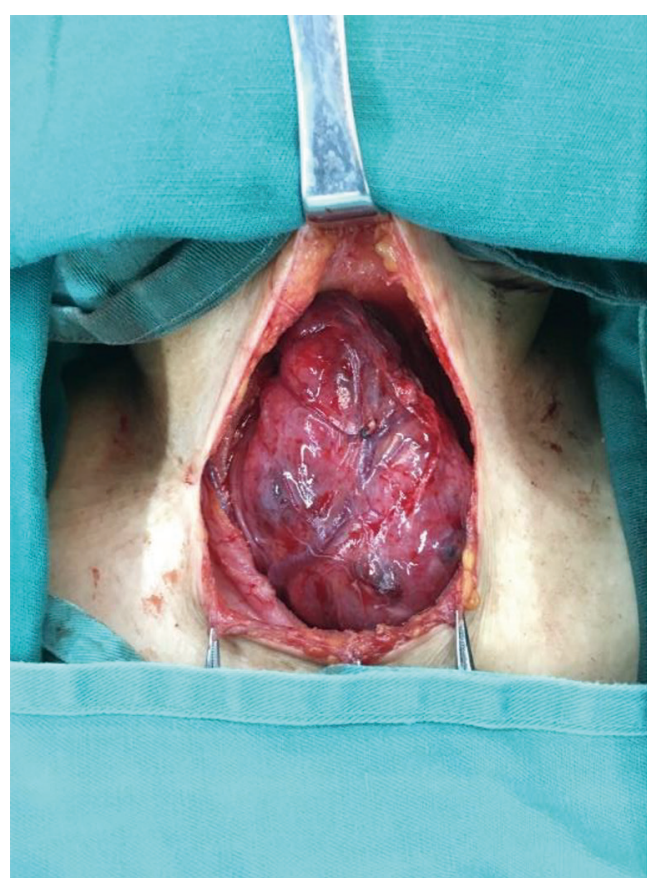

Figure 3 A tumor being removed from the neck.

surgery and by utilizing routine visual nerve identification, the nerve can be protected under direct view. It is also better to use a nerve monitor, which can effectively prevent recurrent laryngeal nerve injury, during the operation. Intraoperative neuromonitoring helps to identify the RLN, especially in difficult cases, and its use can change the operative strategy to prevent the risk of bilateral damage in case of signal loss.

Hypoparathyroidism and hypocalcaemia are the most common complications following thyroid surgery. Surgery for retrosternal goiter means increasing the risk of hypocalcemia due to more difficulty in identifying the parathyroid glands (26). Postoperative hypocalcemia is defined as a serum calcium below $2.11 \mathrm{mmol} / \mathrm{L}$, and hypoparathyroidism is defined as a postoperative serum PTH below $14.5 \mathrm{pg} / \mathrm{mL}$. The incidences of temporary and permanent postoperative hypoparathyroidism range between $7 \%$ to $60 \%$ and $0 \%$ to $9 \%$, respectively, and the incidence of postoperative hypocalcaemia is in the range of $1 \%$ to $50 \%(27,28)$. Recent multicentric analysis showed that the incidence rate of permanent hypocalcaemia was $2.1 \%$ and $2.9 \%$ when cervical and extracervical approaches were adopted, respectively (29). In patients who undergo secondary or even multiple surgery, which can increase adhesion to the surrounding tissue, the risk of hypocalcaemia it is perceived to be higher (29). In this retrospective study, $16(14.3 \%)$ patients had transient hypoparathyroidism. With the cervical and extracervical approaches, hypocalcaemia was transient in 23 patients $(20.5 \%)$ and $2(66.7 \%)$ patients, respectively, and no permanent hypoparathyroidism or hypocalcemia occurred. We recommend that a very accurate dissection should be performed as close to the capsule as possible. When the gland tissue is completely separated from the surrounding tissue, then traction should be applied, and with continued traction and blunt dissection, the mass can be removed (Figure 3).

\section{Conclusions}

Retrosternal goiter surgery is challenging for surgeons. The best surgical approach should be determined based on CT scan evaluation. The complete capsule and the clear boundary between the retrosternal goiter and the surrounding tissue are the most important factors for surgery. During surgery, the shoulder should be raised with the head back to fully expose the surgical field. A collar incision should be made, and blunt separation should be combined with meticulous operation to identify and protect important blood vessels, nerves, and the parathyroid glands. The retrosternal component should then be gradually elevated out of the mediastinum into the neck, and the mass can then be completely removed. The incidence of perioperative complications, such as recurrent laryngeal nerve palsy, hypoparathyroidism, hypocalcemia, and infection, is not significantly different to that seen with traditional thyroid surgery. With careful planning and execution before surgery and meticulous operation during surgery, most retrosternal goiters can be safely removed with cervical surgery.

\section{Acknowledgments}

Funding: None.

\section{Footnote}

Conflicts of Interest: All authors have completed the ICMJE uniform disclosure form (available at http://dx.doi. org/10.21037/gs.2020.03.43). The authors have no conflicts of interest to declare.

Ethical Statement: The authors are accountable for all 
aspects of the work in ensuring that questions related to the accuracy or integrity of any part of the work are appropriately investigated and resolved. The study is not involving any ethical issues and written informed consent was obtained from all patients.

Disclosure: The original data is available upon request.

Open Access Statement: This is an Open Access article distributed in accordance with the Creative Commons Attribution-NonCommercial-NoDerivs 4.0 International License (CC BY-NC-ND 4.0), which permits the noncommercial replication and distribution of the article with the strict proviso that no changes or edits are made and the original work is properly cited (including links to both the formal publication through the relevant DOI and the license). See: https://creativecommons.org/licenses/by-nc-nd/4.0/.

\section{References}

1. Nixon IJ, Simo R, The neoplastic goitre. Curr Opin Otolaryngol Head Neck Surg 2013;21:143-9.

2. Simó R, Nixon IJ, Vander Poorten V, et al. Surgical management of intrathoracic goitres. Eur Arch Otorhinolaryngol 2019;276:305-14.

3. Wang Y, Guan Q, Lao I, et al. Using deep convolutional neural networks for multi-classification of thyroid tumor by histopathology: a large-scale pilot study. Ann Transl Med 2019;7:468.

4. Polistena A, Sanguinetti A, Lucchini R, et al. Surgical approach to mediastinal goiter: An update based on a retrospective cohort study. Int J Surg 2016;28 Suppl 1:S42-6.

5. Mercante G, Gabrielli E, Pedroni C, et al. CT crosssectional imaging classification system for substernal goiter based on risk factors for an extracervical surgical approach. Head Neck 2011;33:792-9.

6. Casella C, Pata G, Cappelli C, et al. Preoperative predictors of sternotomy need in mediastinal goiter management. Head Neck 2010;32:1131-5.

7. Raffaelli M, De Crea C, Ronti S, et al. Substernal goiters: incidence, surgical approach, and complications in a tertiary care referral center. Head Neck 2011;33:1420-5.

8. Nakaya M, Ito A, Mori A, et al. Surgical treatment of substernal goiter: An analysis of 44 cases. Auris Nasus Larynx 2017;44:111-5.

9. Pulli RS, Coniglio JU. Surgical management of the substernal thyroid gland. Laryngoscope 1998;108:358-61.
10. Randolph GW, Shin JJ, Grillo HC, et al. The surgical management of goiter: Part II. Surgical treatment and results. Laryngoscope 2011;121:68-76.

11. Ríos A, Sitges-Serra A. Surgical treatment of intrathoracic goitre. Cir Esp 2012;90:421-8.

12. Lin YS, Wu HY, Lee CW, et al. Surgical management of substernal goitres at a tertiary referral centre: A retrospective cohort study of 2,104 patients. Int J Surg 2016;27:46-52.

13. Qureishi A, Garas G, Tolley N, et al. Can pre-operative computed tomography predict the need for a thoracic approach for removal of retrosternal goitre? Int J Surg 2013;11:203-8.

14. Riffat F, Del Pero MM, Fish B, et al. Radiologically predicting when a sternotomy may be required in the management of retrosternal goiters. Ann Otol Rhinol Laryngol 2013;122:15-9.

15. Grainger J, Saravanappa N, D'Souza A, et al. The surgical approach to retrosternal goiters: the role of computerized tomography. Otolaryngol Head Neck Surg 2005;132:849-51.

16. Tang AL, Falciglia $M$, Yang $H$, et al. Validation of American Thyroid Association Ultrasound Risk Assessment of Thyroid Nodules Selected for Ultrasound Fine-Needle Aspiration. Thyroid 2017;27:1077-82.

17. de Perrot M, Fadel E, Mercier O, et al. Surgical management of mediastinal goiters: when is a sternotomy required? Thorac Cardiovasc Surg 2007;55:39-43.

18. Yan HX, Du J, Fu J, et al. Microarray-based differential expression profiling of long noncoding RNAs and messenger RNAs in formalin-fixed paraffin-embedded human papillary thyroid carcinoma samples. Transl Cancer Res 2019;8:439-51.

19. Cichoń S, Anielski R, Konturek A, et al. Surgical management of mediastinal goiter: risk factors for sternotomy. Langenbecks Arch Surg 2008;393:751-7.

20. Chandrasekhar SS, Randolph GW, Seidman MD, et al. Clinical practice guideline: improving voice outcomes after thyroid surgery. Otolaryngol Head Neck Surg 2013;148:S1-37.

21. Truong P, Dickerson L. An unusual cause of hoarseness and recurrent laryngeal nerve palsy. JAAPA 2016;29:35-7.

22. Shindo ML, Wu JC, Park EE. Surgical anatomy of the recurrent laryngeal nerve revisited. Otolaryngol Head Neck Surg 2005;133:514-9.

23. Vaiman M, Bekerman I, Basel J, et al. Surgical approach to the intrathoracic goiter. Laryngoscope Investig Otolaryngol 2018;3:127-32. 
24. Jeannon JP, Orabi AA, Bruch GA, et al. Diagnosis of recurrent laryngeal nerve palsy after thyroidectomy: a systematic review. Int J Clin Pract 2009;63:624-9.

25. Kilic D, Findikcioglu A, Ekici Y, et al. When is transthoracic approach indicated in retrosternal goiters? Ann Thorac Cardiovasc Surg 2011;17:250-3.

26. Edafe O, Antakia R, Laskar N, et al. Systematic review and meta-analysis of predictors of post-thyroidectomy hypocalcaemia. Br J Surg 2014;101:307-20.

27. Marcinkowska M, Sniecikowska B, Zygmunt A, et al.

Cite this article as: Wang X, Zhou Y, Li C, Cai Y, He T, Sun R, Tian W, Tang Z, Sheng J, Liu D, Gui C, Zeng D, Shui C, Jang J, Zhu G, Ning Y, Wang W. Surgery for retrosternal goiter: cervical approach. Gland Surg 2020;9(2):392-400. doi: 10.21037/ gs.2020.03.43
Postoperative hypoparathyroidism in patients after total thyroidectomy - retrospective analysis. Neuro Endocrinol Lett 2017;38:488-94.

28. Docimo G, Ruggiero R, Casalino G, et al. Risk factors for postoperative hypocalcemia. Updates Surg 2017;69:255-60.

29. Testini M, Gurrado A, Avenia N, et al. Does mediastinal extension of the goiter increase morbidity of total thyroidectomy? A multicenter study of 19,662 patients. Ann Surg Oncol 2011;18:2251-9. 